
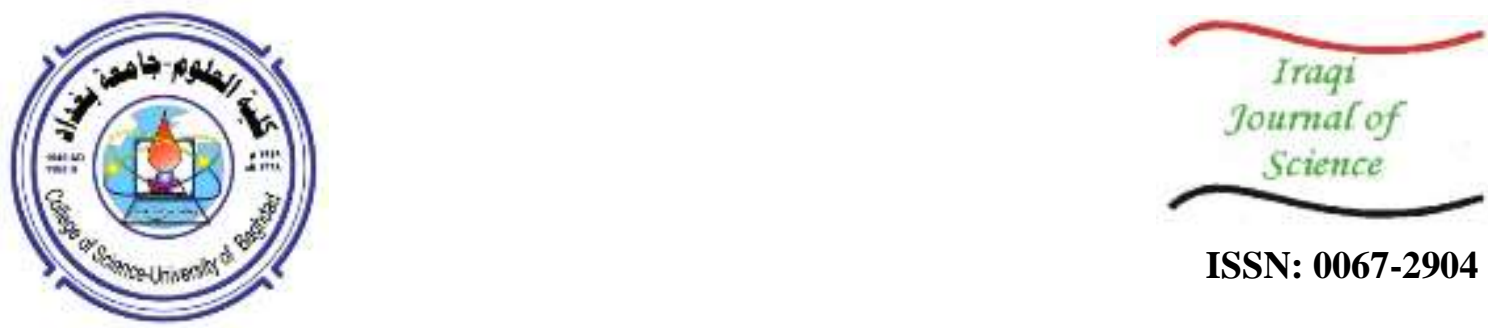

ISSN: 0067-2904

\title{
An Approximation Technique for Fractional Order Delay Differential Equations
}

\author{
Olutunde Samuel Odetunde*, Abass Ishola Taiwo, Olusola Adebanwo Dehinsilu \\ Department of Mathematical Sciences, Olabisi Onabanjo University, Ago-Iwoye, Nigeria
}

\begin{abstract}
In this research article, an Iterative Decomposition Method is applied to approximate linear and non-linear fractional delay differential equation. The method was used to express the solution of a Fractional delay differential equation in the form of a convergent series of infinite terms which can be effortlessly computable. The method requires neither discretization nor linearization. Solutions obtained for some test problems using the proposed method were compared with those obtained from some methods and the exact solutions. The outcomes showed the proposed approach is more efficient and correct.
\end{abstract}

Keywords: Fractional Delay Differential Equation, Caputo Derivative, Iterative Decomposition Method

\section{Introduction}

Fractional Differential Equations (FDE), that are a generalization of the differential equation were applied to model many physical phenomena very appropriately [1]. They have been used drastically in areas like thermal engineering, acoustics, electromagnetism, robotics, viscoelasticity, sign processing and populace dynamics [2]. Delay Differential equations are differential equations involving memory and heritage properties. In real life system, delays can be recognized and thus interest in the study of delay differential has been growing over the years, these include modeling of processes of the system in engineering, sciences and biological systems that involved gestation and maturation as well as population models and mining problems [3].

Although Fractional Delay Differential equations (FDDE) are current, there nevertheless exist a variety of materials in the literature, as an example, [4] described the situations for the existence of the solution of Fractional Delay Differential Equations with Riemann- Liouinlle derivatives. In [5, 6], the existence of positive solution for a class of single - term Fractional Delay Differential Equations are taken into consideration, whilst [6 - 9] considered the steadiness of Fractional Delay Differential Equation. Owing to the increasing interest in the look at FDDE, interest is rapidly moving in the direction of the solution of the equations. Hence, in this research article, Fractional Delay Differential equation of the form

is considered. Subject to the initial situation

$$
\mathrm{D}^{\alpha} \mathrm{y}(x)=f(y)+g(x) y\left(\frac{x}{a}\right), 0<x<b, 0<\propto \leq m
$$

$$
y(0)=\beta_{0}, y^{\prime}(0)=\beta_{1}, y^{\prime \prime}(0)=\beta_{2}, \ldots ., y^{(m)}(0)=\beta_{m}
$$

where $\alpha$ is a proportional, $g(x)$ is a source time period characteristic and $\beta_{i}, i=0,1,2, \ldots$ are actual finite constants.

It is famous that fractional differential equations do no longer have an analytical solution. Several authors have taken into consideration the approximation of Delay Differential Equations of integer order and these consist of the researches of [10 - 14] even as [15-16] taken into consideration the method of splines. [3, 17] implemented the Adomian Decomposition Method (ADM), [18]

*Email: tunde.odetunde@oouagoiwoye.edu.ng 
implemented an Iterative Decomposition Method and [19] used the Differential Transform Method. Fractional Delay Differential equations have been approximated using very many strategies. [20] applied a combination of the trapezoidal and Simpson rule to find a series solution of FDDEs, while [21] implemented the Chebyshev Wavelet approach. Another Wavelet approach, the Hermite Wavelet approach was used by [22]. [23] applied a finite difference - based approach and [24] carried out the Adams - Bashforth - Moulton algorithm. [25] had in advance applied the Homotopy Analysis Method. Then, this research article will be used to implement the application of the Iterative Decomposition Method which has been carried out effectively for integer order differential equations consisting of Delay Differential Equations [18]. The method is devoid of any form of linearization or discretization [1].

\section{Materials and Methods}

There are several definitions of a fractional derivate of order $\propto>0$. Examples include Riemann Liouville, Grunwald - Letnikov, Jumarie, Caputo, Generalised approach derivatives. The most commonly implemented definitions are those of Riemann-Liouville and Caputo. However, the Caputo derivative draws more attention for its ease of adaptability, especially for physical problems. Some relevant basic definitions and relevant properties as they relate to fractional calculus will be mentioned as utilized in [1].

Definition 2.1: A actual characteristic $f(x), x>0$ is stated to be within the space $\mathrm{C}_{\mu}, \mu \in \mathrm{R} \mu$, if there exists a real number $\rho>\mu$, such that $f(x)=x^{P} f(x)$, wherein $f(x) \in C(0, \infty)$, and it is stated to be in space $C_{\mu}^{\mathrm{n}}$ if and only if $f^{(n)} \in \mathrm{C}_{\mu} \mathrm{n} \in \mathbb{N}$. Truly, $C_{\mu} \subset C_{\beta}$ if $\beta \leq \mu$.

Definition 2.2: Let $\propto \geq 0$ and $n<\propto \leq n+1, n \in \mathbb{N}$.

The operator ${ }_{a} D_{t}$, defined by

$$
{ }_{a} \mathrm{D}_{\mathrm{t}} f(t)=\frac{1}{\Gamma(n-\alpha)} \frac{d^{n}}{d t^{n}} \int_{a}^{t}(t-x)^{n-a-1} f(x) d x \quad a \leq t \leq b .
$$

is termed the Riemann - Liouville fractional derivative of order $\propto$.

Definition (2.3): The Riemann - Liouville fractional integral operator defined on $\mathrm{L}_{1}$ [a,b] of order $\propto \geq 0$ of a function $f \in \mathrm{C}_{\mu}, \mu \geq-1$ is described as

$$
\mathrm{J}^{\propto} f(x)=\frac{1}{\Gamma(\alpha)} \int_{0}^{x}(x-t)^{\alpha-1} f(t) d t, \propto>0, x>0
$$

Properties of the operator $\mathcal{J}^{\propto}$ are located in [2] and encompassed the following

$$
\begin{aligned}
& \mathcal{J}^{0} f(x)=f(x) \\
& \mathcal{J}^{\propto} x^{\alpha}=\frac{\Gamma(\Gamma+1)}{\Gamma(\alpha+\Gamma+1)} x^{\propto+r} \\
& \mathcal{J}^{\propto} \mathcal{J}^{\beta} f(x)=\mathcal{J}^{\propto+\beta} f(x) \\
& \mathcal{J}^{\propto} \mathcal{J}^{\beta} f(x)=\mathcal{J}^{\beta} \mathcal{J}^{\alpha} f(x)
\end{aligned}
$$

Definition 2.4: The fractional derivative of the function $f(x)$ in the Caputo sense is described as

$$
D_{*}^{\propto} f(x)=\mathcal{J}^{m-\propto} D^{m} f(x)=\frac{1}{\Gamma(m-\alpha)} \int_{0}^{x}(x-t)^{m-\alpha-1} f^{(m)}(t) d t
$$

For $m-1<\propto<m ; m \epsilon \mathbb{N}$ and $f \epsilon \mathrm{C}_{\mu}^{m}, \mu \geq-1$.

Lemma 2.1: If $m-1<\propto<m, m \in i N$ and $f \in \mathrm{C}_{\mu}^{m}, \mu z-1$. then;

\section{The Decomposition method}

$$
\begin{aligned}
\mathrm{D}_{*}^{\alpha} \mathcal{J}^{\propto} f(x) & =f(x) \\
\mathcal{J}^{\propto} \mathrm{D}_{*}^{\alpha} f(x) & =f(x)-\sum_{k=0}^{m-1} \frac{x^{k}}{k !} f^{(k)}(0), x \leq 0
\end{aligned}
$$

The utilization of the IDM [1] to FDDE will be assessed. Applying the inverse operator in equation (4) to both sides of equation (1) to attain

$$
y(x)=\sum_{k=0}^{m-1} \frac{x^{k}}{k !} y^{(k)}(0)+\mathcal{J}^{\propto}\left\{f(x)+g(x) y\left(\frac{x}{a}\right)\right\}
$$

By the IDM, the solution $y(x)$ could be spilted into the infinite series of convergent terms

for every $n=1,2 \ldots$

$$
y(x)=\sum_{n=0}^{\infty} y_{n}(x)
$$

The Iterative Decomposition method suggests that the non-linear terms could be decomposed as

$$
N\left(\sum_{n=0}^{\infty} y_{n}\right)=N\left(y_{0}\right)+\sum_{j=1}^{\infty}\left\{N\left(\sum_{j=0}^{n} y_{j}\right)-N\left(\sum_{j=0}^{n-1} y_{j}\right)\right\}
$$

where $\mathrm{N}$ is a non-linear operator, which in this situation may be replaced by the Riemann - Liouville integral operator in equation (4).

From equations (13) and (14) 
and

$$
\sum_{n=0}^{\infty} y_{n}=\beta_{1}+N\left(y_{0}\right)+\sum_{j=1}^{\infty}\left\{N\left(\sum_{j=0}^{n} y_{j}\right)-N\left(\sum_{j=0}^{n-1} y_{j}\right)\right\}
$$

$$
\left.\begin{array}{c}
y_{0}=y(0) \\
y_{1}=N\left(y_{0}\right) \\
y_{2}=N\left(y_{0}+y_{1}\right)-N\left(y_{0}\right) \\
\vdots \\
y_{n+1}=N\left(y_{0}+y_{1} \ldots+y_{n}\right)-N\left(y_{0}+y_{1} \ldots+y_{n+1}\right)
\end{array}\right\}
$$

Then solution in (13) is an approximation from (16).

\section{Results and Discussion}

\section{Numerical Examples}

Some numerical examples will be illustrated to decide the adaptability of the postulated approach.

\section{Example 1:}

Consider the Fractional Delay Differential equation

$$
D_{*}^{\alpha} y(x)-y\left(\frac{x}{2}\right)=0,0<\propto \leq 1, x>0
$$

Subject to the preliminary condition

$$
y(0)=1
$$

The exact solution for $\propto=1$ is

$$
y(x)=\sum_{k=0}^{\infty} \frac{\left(\frac{1}{2}\right)^{k(k-1)}}{k !} x^{k}
$$

Using the inverse operator on both sides of (17),

$$
y(x)=1+\mathrm{J}^{\propto}\left\{y\left(\frac{x}{2}\right)\right\}
$$

Taking $y_{0}=1$ to attain

$$
\begin{aligned}
y_{1} & =\frac{x^{\propto}}{\Gamma(\alpha+1)} \\
y_{2} & =\frac{x^{\propto}}{\Gamma(\alpha+1)}+\frac{x^{2 \alpha}}{2^{\alpha} \Gamma(2 \alpha+1)}-\frac{x^{\alpha}}{\Gamma(\propto+1)} \\
y_{2} & =\frac{x^{2 \alpha}}{2^{\alpha} \Gamma(2 \alpha+1)} \\
y_{3} & =\frac{x^{3 \alpha}}{2^{3 \alpha} \Gamma(3 \alpha+1)} \\
y_{4} & =\frac{x^{4 \alpha}}{2^{6 \alpha} \Gamma(4 \alpha+1)} \\
y_{5} & =\frac{x^{5 \propto}}{2^{10 \propto} \Gamma(5 \alpha+1)} \\
y_{6} & =\frac{x^{6 \propto}}{2^{15 \alpha} \Gamma(6 \propto+1)}
\end{aligned}
$$

$$
y(x)=1+\frac{x^{\alpha}}{\Gamma(\alpha+1)}+\frac{x^{2 \alpha}}{2^{\alpha} \Gamma(2 \alpha+1)}+\frac{x^{3 \alpha}}{2^{3 \alpha} \Gamma(3 \alpha+1)}+\frac{x^{4 \alpha}}{2^{6 \alpha} \Gamma(4 \alpha+1)}+\frac{x^{5 \alpha}}{2^{10 \alpha} \Gamma(5 \alpha+1)}+\ldots
$$

for $\propto=1$, equation (27) becomes

$$
y(x)=1+x+\frac{x^{2}}{2 \cdot 2 !}+\frac{x^{3}}{2^{3} \cdot 3 !}+\frac{x^{4}}{2^{6} \cdot 4 !}+\frac{x^{5}}{2^{10} \cdot 5 !}+\frac{x^{6 \propto}}{2^{15 \alpha} \Gamma(6 \propto+1)}+\cdots
$$

Table 1- Approximate Solution of Example 1 at $\propto=2$ and comparison with exact and CWM solution

\begin{tabular}{|c|c|c|c|c|c|}
\hline $\mathbf{X}$ & Exact & IDM solution & Error of IDM & $\begin{array}{c}\text { CWM } \\
\text { solution }\end{array}$ & Error of CWM \\
\hline 0.00 & 1.000000000 & 1.00000000 & 0.00000000 & 11.00000000 & $4.000000 \times 10^{-10}$ \\
\hline 0.01 & 1.010012503 & 1.010025021 & $1.2518 \times 10^{-5}$ & 1.01002844 & $3.42200 \times 10^{-6}$ \\
\hline 0.02 & 1.020050021 & 1.020100167 & $5.0146 \times 10^{-5}$ & 1.02010706 & $6.89300 \times 10^{-6}$ \\
\hline 0.03 & 1.030112570 & 1.030225563 & $1.12993 \times 10^{-4}$ & 1.03023597 & $1.04100 \times 10^{-5}$ \\
\hline 0.04 & 1.040200167 & 1.040401335 & $2.01168 \times 10^{-4}$ & 1.04041530 & $1.3965 \times 10^{-5}$ \\
\hline 0.05 & 1.050312826 & 1.050627608 & $3.14782 \times 10^{-4}$ & 1.05064516 & $1.75560 \times 10^{-5}$ \\
\hline 0.06 & 1.060450563 & 1.060904508 & $4.53945 \times 10^{-4}$ & 1.06092568 & $2.11750 \times 10^{-5}$ \\
\hline 0.07 & 1.070613393 & 1.071232161 & $6.18768 \times 10^{-4}$ & 1.07125698 & $2.48180 \times 10^{-5}$ \\
\hline
\end{tabular}




\begin{tabular}{|l|c|c|c|c|c|}
\hline 0.08 & 1.080801334 & 1.081610693 & $8.09359 \times 10^{-4}$ & 1.08163917 & $2.84790 \times 10^{-5}$ \\
\hline 0.09 & 1.091014399 & 1.09204023 & $1.025831 \times 10^{-3}$ & 1.09207238 & $3.21530 \times 10^{-5}$ \\
\hline 0.10 & 1.101252605 & 1.102520898 & $1.268293 \times 10^{-3}$ & 1.10255673 & $3.58360 \times 10^{-5}$ \\
\hline
\end{tabular}

\section{Example 2}

Consider the second order nonlinear FDDE

$$
D^{\alpha} \mathrm{y}(\mathrm{x})=1-2 \mathrm{y}^{2}\left(\frac{x}{2}\right), 0 \leq x \leq 1, \quad 1<\propto \leq 2,
$$

subjected to the preliminary conditions

$$
\mathrm{y}(0)=1, y^{\prime}(0)=0
$$

The exact solution of the problem at $\propto=2$ is

Using the inverse operator on (31)

$$
\mathrm{y}(x)=\cos x
$$

$$
\mathrm{y}(x)=1+\frac{x^{\alpha}}{\Gamma(\alpha+1)}-J^{\alpha} f(x)=\left\{2 y^{2}\left(\frac{x}{2}\right)\right\}
$$

Taking $\mathrm{y}_{\mathrm{o}}=1+\frac{x^{\alpha}}{\Gamma(\alpha+1)^{\prime}}$

Then from equation (31) by way of IDM

$$
\begin{aligned}
& \mathrm{y}_{1}=-\left\{\frac{2 x^{\alpha}}{\Gamma(\alpha+1)}+\frac{x^{2 \alpha}}{2^{\alpha-2} \Gamma(2 \alpha+1)}+\frac{\Gamma(2 \alpha+1) X^{3 \alpha}}{2^{2 \alpha-1}\left[\Gamma(\alpha+1) J^{2} \Gamma(3 \alpha+1)\right]}\right\} \\
& \mathrm{y}_{2}=-\left.\frac{x^{2 \alpha}}{2^{\alpha-3} \Gamma(2 \alpha+1)}+\frac{x^{3 \alpha}}{2^{3 \alpha-4} \Gamma(3 \alpha+1)}-\frac{\Gamma(3 \alpha+1) x^{4 \propto}}{2^{4 \alpha-4}[\Gamma(\alpha+1) \Gamma(2 \alpha+1) \Gamma(4 \alpha+1)]}\right\} \\
&+\frac{\Gamma\left(2 x^{\alpha}+1\right) x^{4 \alpha}}{2^{5 \alpha-3}\left[\Gamma(\alpha+1) J^{2} \Gamma(4 \propto+1)\right]}-\frac{\Gamma(2 \alpha+\alpha 1) \Gamma(4 \alpha+1) x^{5 \alpha}}{2^{6 \alpha-3}\left[\Gamma(\alpha+1) J^{3} \Gamma(3 \alpha+1) r(5 \alpha+1)\right]} \\
&-\frac{\Gamma\left(4^{\alpha}+1\right) x^{4 \alpha}}{2^{6 \alpha-5}\left[\Gamma(2 \alpha+1) J^{2} \Gamma(5 \alpha+1)\right]}-\frac{\Gamma(2 \alpha+1) \Gamma(5 \alpha+1) x^{6 \alpha}}{2^{8 \alpha-5} \Gamma\left[(\alpha+1)^{2} \Gamma(2 \alpha+1) \Gamma(3 \alpha+1) \Gamma(6 \alpha+1)\right]} \\
&-\frac{\left[\Gamma\left(2^{\alpha}+1\right)^{2} \Gamma(6 \alpha+1) x^{7 \alpha}\right]}{2^{10 \alpha-3}\left[\Gamma(\alpha+1) J^{4}[\Gamma(3 \alpha+1)]^{2} \Gamma(7 \alpha+1)\right]}+\ldots
\end{aligned}
$$

Then $\mathrm{y}(\mathrm{x})$ may be approximated as

$$
\begin{aligned}
& y(x)=1-\frac{x^{\propto}}{\Gamma(\propto+1)}+\frac{x^{2 \propto}}{2^{\alpha-2} \Gamma(2 \propto+1)}-\frac{r(2 \propto+1) x^{3 \propto}}{2^{2 \alpha-1}\left[\Gamma(\propto+1)^{2} \Gamma(3 \propto+1)\right]} \\
& +\frac{x^{3 \alpha}}{2^{3 \alpha-4} \Gamma(3 \alpha+1)}+\frac{\Gamma(3 \alpha+1) x^{4 \alpha}}{2^{4 \alpha-4} \Gamma(\alpha+1) \Gamma(2 \alpha+1) \Gamma(4 \alpha+1)}+\frac{\Gamma(2 \alpha+1) x^{4 \alpha}}{2^{5 \alpha-3}\left[\Gamma(\alpha+1)^{2} \Gamma(4 \alpha+1)\right]} \\
& -\frac{\Gamma(2 \alpha+1) \Gamma(4 \alpha+1) x^{5 \alpha}}{2^{6 \alpha-3}\left[\Gamma(\alpha+1)^{3} \Gamma(3 \alpha+1) \Gamma(5 \alpha+1)\right]}-\frac{\Gamma(4 \propto+1) x^{5 \propto}}{2^{6 \alpha-5}\left[\Gamma(2 \alpha+1)^{2} \Gamma(5 \propto+1)\right]} \\
& -\frac{\Gamma(2 \alpha+1) \Gamma(5 \alpha+1) x^{6 \propto}}{2^{8 \alpha-5}\left[\Gamma(\alpha+1)^{2} \Gamma(2 \alpha+1) \Gamma(2 \alpha+1) \Gamma(3 \alpha+1) \Gamma(6 \alpha+1)\right]}-\frac{\left[\Gamma(2 \alpha+1)^{2} \Gamma(6 \alpha+1) x^{7 \alpha}\right.}{2^{10 \alpha-3}\left[\Gamma(\alpha+1)^{4}\left[\Gamma(3 \alpha+1)^{2} \Gamma(7 \alpha+1)\right]\right.}
\end{aligned}
$$

For the particular case $\propto=2$,

$$
y(x)=1-\frac{x^{2}}{2}+\frac{x^{4}}{4 !}-\frac{x^{6}}{6 !}+\frac{x^{8}}{8 !}-\frac{x^{10}}{10 !}+\frac{x^{12}}{12 !}-\frac{x^{14}}{14 !}+\frac{x^{16}}{16 !}-\frac{x^{18}}{18 !} . .
$$

Table 2 - Approximate Solution of Example 2 at $\propto=2$ and comparison with exact and CWM solution

\begin{tabular}{|c|c|c|c|c|c|}
\hline $\mathbf{X}$ & Exact & IDM solution & Error & $\mathbf{y}_{\mathbf{2}}$ & Error $\mathbf{y}_{\mathbf{2}}$ \\
\hline 0.00 & 1.000000000 & 1.00000000 & 0.00000000 & -0.00000000 & $1.00000 \times 10^{-10}$ \\
\hline 0.01 & 0.9999500004 & 0.9999541667 & $4.166266665 \times 10^{-6}$ & 0.00010000 & $1.00000 \times 10^{-10}$ \\
\hline 0.02 & 0.9998000067 & 0.9998000067 & $-3.34222000 \times 10^{-11}$ & 0.00040000 & $1.00000 \times 10^{-10}$ \\
\hline 0.03 & 0.9995500337 & 0.9995500337 & $4.89875000 \times 10^{-11}$ & 0.00900000 & $1.00000 \times 10^{-10}$ \\
\hline 0.04 & 0.9992001067 & 0.9992001067 & $-3.902210000 \times 10^{-11}$ & 0.00160000 & $1.00000 \times 10^{-10}$ \\
\hline 0.05 & 0.9987502604 & 0.9987502604 & $-5.033800000 \times 10^{-12}$ & 0.00250000 & $1.00000 \times 10^{-10}$ \\
\hline 0.06 & 0.9982005399 & 0.9982005399 & $3.520410000 \times 10^{-11}$ & 0.00360000 & $1.00000 \times 10^{-10}$ \\
\hline 0.07 & 0.9975510003 & 0.9975510003 & $-4.672050000 \times 10^{-11}$ & 0.00490000 & $1.00000 \times 10^{-10}$ \\
\hline 0.08 & 0.9968017063 & 0.9968017063 & $2.619400000 \times 10^{-12}$ & 0.00640000 & $1.00000 \times 10^{-10}$ \\
\hline 0.09 & 0.9959527330 & 0.9959527330 & $1.199420000 \times 10^{-11}$ & 0.00810000 & $1.00000 \times 10^{-10}$ \\
\hline 0.10 & 0.9950041653 & 0.9999500004 & $4.94583511 \times 10^{-3}$ & 0.01000000 & $1.00000 \times 10^{-10}$ \\
\hline
\end{tabular}




\section{Example 3}

Consider the FDDE

$$
D_{*}^{\alpha} y(x)=\frac{3}{4} y(x)+y\left(\frac{x}{2}\right)-x^{2}+2,0 \leq x \leq 1,1<\propto \leq 2
$$

Subject to the preliminary condition

$$
y(0)=0
$$

The exact solution is given as $y(x)=x^{2}$ for $\propto=2$. By the IDM,

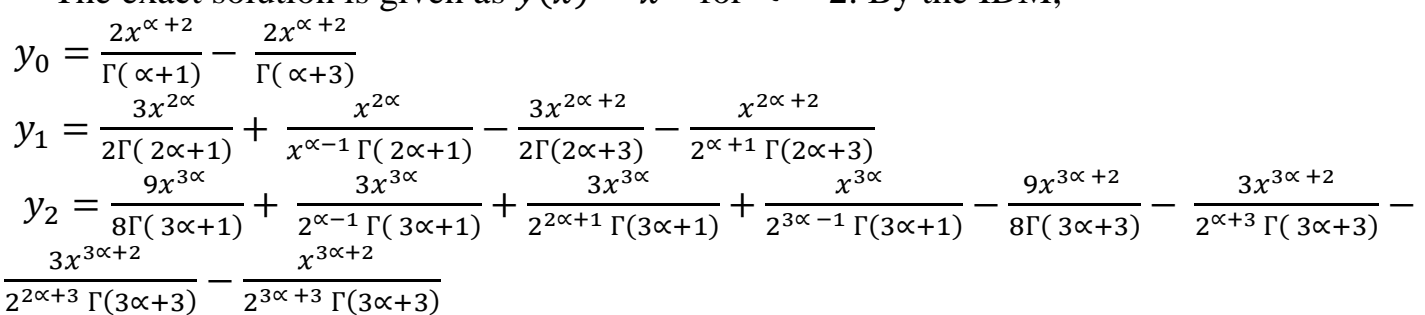

Then, by approximation

$$
\begin{aligned}
& y(x)=\frac{2 x^{\alpha}}{\Gamma(\alpha+1)}+\frac{3 x^{2 \alpha}}{2 \Gamma(2 \propto+1)}+\frac{x^{2 \alpha}}{2^{\alpha-1} \Gamma(2 \alpha+1)}+\frac{9 x^{3 \alpha}}{8 \Gamma(3 \alpha+1)}+\frac{3 x^{3 \alpha}}{2^{\alpha+1} \Gamma(3 \alpha+1)}+\frac{3 x^{3 \alpha}}{2^{\alpha+1} \Gamma(3 \alpha+1)}+\frac{x^{3 \alpha}}{2^{3 \alpha-1 \Gamma(33 \alpha-1)}}- \\
& \frac{2 x^{\alpha+2}}{\Gamma(\alpha+3)}-\frac{3 x^{2 \alpha+2}}{2 \Gamma(2 \alpha+3)} \\
& \quad-\frac{x^{2 \alpha+2}}{2^{2 \alpha+1} \Gamma(2 \alpha+3)}-\frac{9 x^{3 \alpha+2}}{8 \Gamma(3 \alpha+3)}-\frac{3 x^{3 \alpha+2}}{2^{\alpha+3} \Gamma(3 \propto+3)}-\frac{3 x^{3 \alpha+2}}{2^{2 \alpha+3} \Gamma(3 \propto+3)}-\frac{x^{3 \alpha+2}}{2^{3 \alpha+3} \Gamma(3 \alpha+3)}
\end{aligned}
$$

For the particular case $\propto=2$,

$$
y(x)=x^{2}-\frac{319 x^{8}}{10321920}
$$

Table 3- Approximate solution of Example 3 at $\propto=2$ and Comparison with exact and CWM solution

\begin{tabular}{|c|c|c|c|c|c|}
\hline $\boldsymbol{X}$ & Exact Solution & IDM Solution & Error of IDM & CWM Solution & Error of CWM \\
\hline 0.00 & 0.0000000 & 0.0000000 & 0.000000 & 0.000000 & $1.00000 \times 10^{-10}$ \\
\hline 0.01 & 0.00010000 & 0.0001000000 & $1.000000 \times 10^{-10}$ & 0.00010000 & $1.00000 \times 10^{-10}$ \\
\hline 0.02 & 0.00040000 & 0.000400000 & $1.00000 \times 10^{-10}$ & 0.00040000 & $1.00000 \times 10^{-10}$ \\
\hline 0.03 & 0.00090000 & 0.000900000 & $1.00000 \times 10^{-10}$ & 0.00090000 & $1.00000 \times 10^{-10}$ \\
\hline 0.04 & 0.00160000 & 0.00160000 & $1.00000 \times 10^{-10}$ & 0.00160000 & $1.00000 \times 10^{-10}$ \\
\hline 0.05 & 0.00250000 & 0.00250000 & $1.20700 \times 10^{-15}$ & 0.00250000 & $1.00000 \times 10^{-10}$ \\
\hline 0.06 & 0.00360000 & 0.003600000 & $5.19000 \times 10^{-15}$ & 0.00360000 & $1.00000 \times 10^{-10}$ \\
\hline 0.07 & 0.00490000 & 0.004900000 & $1.78160 \times 10^{-15}$ & 0.00490000 & $1.00000 \times 10^{-10}$ \\
\hline 0.08 & 0.00640000 & 0.006400000 & $5.18500 \times 10^{-14}$ & 0.00640000 & $1.00000 \times 10^{-10}$ \\
\hline 0.09 & 0.00810000 & 0.008100000 & $1.33036 \times 10^{-13}$ & 0.00810000 & $1.00000 \times 10^{-10}$ \\
\hline 0.10 & 0.001000000 & 0.010000000 & $3.0905 \times 10^{-13}$ & 0.01000000 & $1.00000 \times 10^{-10}$ \\
\hline
\end{tabular}

\section{Example 4}

Consider the FDDE

$$
D_{*}^{\propto} y(x)=\frac{1}{2} e^{x / 2} y\left(\frac{x}{2}\right)+\frac{1}{2} y(x), 0<\propto \leq 1
$$

Subject to the preliminary condition: $y(x)=1$

The exact solution for $\propto=1$ is $y(x)=e^{x}$

By the IDM, $y_{0}=1$

$$
\begin{aligned}
& y_{1}=\frac{x^{\alpha}}{\Gamma(\alpha+1)}+\frac{x^{\alpha+1}}{4 \Gamma(\alpha+2)}+\frac{x^{\alpha+1}}{8 \Gamma(\alpha+3)} \\
& y_{2}=\frac{x^{2 \alpha}}{2^{\alpha+1} \Gamma(2 \alpha+1)}+\frac{\Gamma(\alpha+2) x^{2 \alpha+1}}{x^{2 \alpha+2} \Gamma(\alpha+1) \Gamma(2 \alpha+2)}+\frac{x^{2 \alpha+1}}{x^{\alpha+4} \Gamma(2 \alpha+2)} \quad+\frac{\Gamma(\alpha+3) x^{2 \alpha+2}}{2^{\alpha+4} \Gamma(\alpha+1) \Gamma(2 \alpha+3)}+\frac{x^{2 \alpha+2}}{16 \Gamma(2 \alpha+3)}+ \\
& \frac{\Gamma(\alpha+3) x^{2 \alpha+2}}{2^{\alpha+5} \Gamma(\alpha+2) \Gamma(2 \propto+3)}+\frac{\Gamma(\alpha+4) x^{2 \alpha+3}}{2^{\alpha+7} \Gamma(\alpha+2) \Gamma(2 \alpha+4)}+\frac{\Gamma(\alpha+4) x^{2 \alpha+3}}{32 \Gamma(\alpha+3) \Gamma(2 \propto+4)}+\frac{\Gamma(\alpha+5) x^{2 \alpha+4}}{128 \Gamma(\alpha+3) \Gamma(2 \propto+5)} \quad+\frac{x^{2 \alpha}}{2 \Gamma(2 \alpha+1)}+ \\
& \frac{x^{2 \propto+1}}{8 \Gamma(2 \propto+2)}+\frac{x^{2 \alpha+2}}{16 \Gamma(2 \propto+3)}
\end{aligned}
$$


Then the approximate solution is

$$
\begin{aligned}
& y(x)=1+\frac{x^{\alpha}}{\Gamma(\alpha+1)}+\frac{x^{\alpha+1}}{4 \Gamma(\alpha+2)}+\frac{x^{\alpha+2}}{8 \Gamma(\alpha+3)}+\frac{x^{2 \alpha}}{2 \Gamma(2 \alpha+1)}+\frac{x^{2 \alpha}}{2^{\alpha+1} \Gamma(2 \alpha+1)} \\
& +\frac{x^{2 \alpha+1}}{8 \Gamma(2 \alpha+2)}+\frac{r(\alpha+2) x^{2 \alpha+1}}{2^{\alpha+2} \Gamma(\alpha+1) \Gamma(2 \alpha+2)}+\frac{x^{2 \alpha+1}}{2^{\alpha+4} \Gamma(2 \alpha+2)}+\frac{x^{2 \alpha+2}}{8 \Gamma(2 \alpha+3)} \\
& +\frac{\Gamma(\alpha+3) x^{2 \alpha+2}}{x^{\alpha+4} \Gamma(\alpha+1) \Gamma(2 \alpha+3)}+\frac{\Gamma(\alpha+3) x^{2 \alpha+2}}{2^{\alpha+5} \Gamma(\alpha+2) \Gamma(2 \alpha+3)}+\frac{\Gamma(\alpha+4) x^{2 \alpha+3}}{32 \Gamma(\alpha+3) \Gamma(2 \alpha+4)} \\
& +\frac{\Gamma(\alpha+4) x^{2 \alpha+3}}{2^{\alpha+7} \Gamma(\alpha+2) \Gamma(2 \alpha+4)}+\frac{\Gamma(\alpha+5) x^{2 \alpha+4}}{128 \Gamma(\alpha+3) \Gamma(2 \alpha+5)}
\end{aligned}
$$

For the particular case $\propto=1$

$$
y(x)=1+x+\frac{x^{2}}{2 !}+\frac{x^{3}}{3 !}+\frac{x^{4}}{4 !}+\frac{x^{5}}{5 !}+\frac{x^{6}}{6 !}+\ldots
$$

Table 4- Numerical Results for Example. 4 for $\propto=1$

\begin{tabular}{|l|c|c|c|c|c|}
\hline $\boldsymbol{X}$ & Exact Solution & IDM Solution & Error IDM & $\begin{array}{c}\text { CWM } \\
\text { Solution }\end{array}$ & Error of CWM \\
\hline 0.00 & 1.00000000 & 1.0000000 & 0.0000000 & 1.00000000 & 0.00000000 \\
\hline 0.01 & 1.01005017 & 1.010050167 & $2.915834 \times 10^{-9}$ & 1.01023273 & $1.82567 \times 10^{-4}$ \\
\hline 0.02 & 1.02020134 & 1.02020134 & $2.6753 \times 10^{-11}$ & 1.02056979 & $3.68446 \times 10^{-4}$ \\
\hline 0.03 & 1.03045453 & 1.030454534 & $3.953512 \times 10^{-9}$ & 1.03101194 & $5.57408 \times 10^{-4}$ \\
\hline 0.04 & 1.04081077 & 1.040810774 & $4.192353 \times 10^{-9}$ & 1.04155999 & $7.49213 \times 10^{-4}$ \\
\hline 0.05 & 1.05127110 & 1.051271096 & $3.624134 \times 10^{-9}$ & 1.05221470 & $9.43609 \times 10^{-4}$ \\
\hline 0.06 & 1.06183655 & 1.061836547 & $3.4552 \times 10^{-9}$ & 1.06297688 & $1.14034 \times 10^{-3}$ \\
\hline 0.07 & 1.07250818 & 1.072508181 & $1.252566 \times 10^{-9}$ & 1.07384730 & $1.33912 \times 10^{-3}$ \\
\hline 0.08 & 1.08328708 & 1.083287068 & $2.329247 \times 10^{-9}$ & 1.08482676 & $1.53969 \times 10^{-3}$ \\
\hline 0.09 & 1.09417428 & 1.094174284 & $3.695612 \times 10^{-9}$ & 1.09591602 & $1.74174 \times 10^{-3}$ \\
\hline 0.10 & 1.10517092 & 1.105170918 & $1.944447 \times 10^{-9}$ & 1.10711589 & $1.94497 \times 10^{-3}$ \\
\hline
\end{tabular}

\section{Conclusion}

A numerical algorithm termed the Iterative Decomposition Method has been carried out to resolve linear and nonlinear Fractional Delay Differential. The result compared favorably with other previous outcomes from acknowledged approaches. The results obtained suggest that the IDM is a method that can be used to obtain solutions of fractional DDE's even when the exact solutions are unknown.

\section{References}

1. Odetunde, O.S. and Taiwo, O. A. 2014. A Decomposition Algorithm for the Solution of Fractional Quadratic Riccati Differential Equations with Caputo Derivatives. American Journal of Computational and Applied Mathematics, 4(3): 83-91.

2. Podlubny, I. 1999. Fractional Differential Equations. Academic Press, San Diego.

3. Adomian, G. 1994. Solving Frontiers Problems of Physics: The Decomposition Method (First Ed.) Reliver Academic, Boston.

4. Lakshmikantham, V. 2008. Theory of Fractional Functional Differential Equations. Journal of Nonlinear Science, 69(10): 3337 - 3343.

5. Ye, H., Ding, Y. and Gao, J. 2007. The existence of a positive solution of $D^{\propto}[x(t) x(0)] x(t) f\left(t, \mathrm{x}_{\mathrm{t}}\right)$. Positivity, Springer Link, 11:341 - 350 .

6. Liao C. and Ye H. 2009. Existence of positive solutions of nonlinear fractional delay differential Equations. Positivity, 13: 601-609.

7. Chen, Y. and Moore, K. L, 2002. Analytical Stability bound for a class of Delayed Fractional Order Dynamic Systems. Nonlinear Dynamics, 29: 191 -200.

8. Deng, W. L. and Lee, J. 2007. Stability of Linear Fractional Differential System with Multiple time delays. Nonlinear Dynamic, 48: 409-416.

9. Krol, k. 2011. Asymptotic Properties of Fractional Delay Differential Equation. Applied Mathematics and Computer, 21(5): 1515-1532.

10. El-Saffy, A. and Abo-Hasha, S. M. 1990. On the Application of Spline Functions to Initial Value 
Problem with Retarded Argument. International Journal of Computational Mathematics, 32(3): $173-179$.

11. El-Safty, A. 1993. Approximate Solution of the Delay Differential Equation $y^{\prime \prime} f(x, y(x), y((x))$ with cubic spline functions. Bulletin of Faulty. Science, Assuit University, 22: $67-73$.

12. Ibrahim, M. A. K., El-Safty, A., Abo-Hasha, S. M. 1995. 2h-Step Spline Method for the Solution of Delay Differential Equations. Computer Mathematics with Applications, 29(8): 1-6.

13. Adomian, G. and Rach, R. 1993. Analytic Solution of Nonlinear Boundary Value Problem in Several Dimensions by Decomposition. Journal of Mathematical Analysis and Application, 174: $118-137$.

14. Wazwaz, A. M. 2000. Approximate Solutions to Boundary Value Problems of Higher Order by the Modified Decomposition Method. International Journal of Computer and Mathematics with Applications, 40: 679-691.

15. Taiwo, O. A. and Ogunlaran, O. M. 2008. Numerical Solution of Fourth Order Linear Ordinary Differential Equations by Cubic Spline Collocation tau Method. Journal of Mathematics and Statistics, 4(4): 264-268.

16. Taiwo, O.A. and Odetunde, O. S. 2009. Approximate Solution of Variational Problems by an Iterative Decomposition Method. Maejo International Journal of Science and Technology, 3(3):426-433.

17. Evans, D. J. and Raslan, K. R. 2005. The Adomian Decomposition Method for Solving DelayDifferential Equations, International Journal of Computational Mathematics, 8: 1 - 6.

18. Taiwo, O.A. and Odetunde, O. S. 2010. On the Numerical Approximation of Delay Differential Equation by a Decomposition Method. Asian Journal Mathematics and Statistics, 3(4): 237- 243.

19. Karako, F. and Bereketoglu, H. 2009, Solution of Delay Differential Equations by Using Differential Transform Method. International Journal of Computer Mathematics, 86: 914-923.

20. Farshad, M. K. 2011. Low-cost Numerical Algorithm to find the Series Solution of Nonlinear Fractional Differential Equations with Delay. Procedia Computer Science, 3: 227-231.

21. Iqbal, M.A, Ali, A. and Mohywd - Din, S. T. 2013. Chebyshev Wavelet Method for Fractional Delay Differential Equations, International Journal of Modern Applied Physics, 4(1): 49-61.

22. Saeed, U. and Rehman, M. 2014. Hermite Wavelet Method for Fractional Delay Differential Equations. Journal of Difference Equations, :1 - 8 .

23. Moghaddam, B. P. and Mostaghim, Z. S. 2013. A numerical method based on Finite Difference for Solving Fractional Delay Differential Equations, Journal of Taibah University for Science, 7: 120 $-127$.

24. Sweilam, N. H., Nagy, A. M., Assiri, T. A. and Ali, N. Y. 2015. Numerical Simulation for Variable - Order Fractional Non-linear Delay Differential Equations, Journal of Fractional Calculus and Applications, 6(1): $71-82$.

25. Mohammed, O. H. and Khlaif, A. I. 2014. Homotopy Analysis for Solving Delay Differential Equations of Fractional Order. Mathematical Theory and Modeling, 4(14): 48 - 56. 